5 Oram M. Efficacy and enlightenment: LSD psychotherapy and the drug amendments of 1962. J Hist Med Allied Sci 2012; 69: 221-50.

6 Hoffer A, Osmond H. The Hallucinogens. Academic Press, 1968.

Matthew M. Nour, CT1 in psychiatry, Jacob Krzanowski, CT1 in psychiatry, South London and Maudsley NHS Foundation Trust, London, UK. Email: matthewnour@ slam.nhs.uk

doi: $10.1192 / \mathrm{bjp} .206 .5 .433 a$

Author's reply: MDMA research is a fascinating branch of research medicine that is now really taking off. Dr Pathania refers to the recent work of Mithoefer and colleagues, whose long-term follow-up study showed a sustained absence of PTSD symptoms in 20 patients with treatment-resistant PTSD 4 years after a single course of MDMA-assisted psychotherapy.

In the wake of these pilot studies, MDMA therapy research is now moving into phase 3 , with large, multicentre trials beginning within the next 24 months (see www.maps.org/research/mdma for more details). This includes, we hope, a UK-based arm of the project and a planned licensing date for MDMA as a prescription medicine for treatment-resistant PTSD by 2021. These are bold steps indeed. For the large population of patients with PTSD who remain chronically unwell and untreated by traditional methods (almost $50 \%$ of all sufferers) this cannot come soon enough.

Drs Nour \& Krzanowski provided a thoughtful and stimulating reply to the article I co-authored with Dr Matt Johnson regarding the contemporary development of psychedelic drug-assisted psychotherapy for drug dependence disorders. ${ }^{1}$ They are absolutely correct to draw attention to the importance of set and setting. These are essential factors to bear in mind whenever a psychedelic drug is used - either clinically, during research or recreationally; the outcome of a psychedelic experience is highly dependent on the user's mindset and the environmental conditions in which they take the drug. ${ }^{2}$ All the research studies Dr Johnson and I mentioned in our review have appropriately paid attention to the concepts of set and setting.

In Dr Johnson's work within the USA with psilocybin, in all the UK-based psychedelic drug studies that I have contributed towards in recent years (with LSD, ketamine and psilocybin), and in our forthcoming UK-based MDMA study, we have been careful to ensure that participants are fully informed about the drugs they are taking, that appropriate safety measures are in place to reassure them and that the studies are conducted in safe, welcoming, relaxed and facilitative environments. These measures are an important active part of the drug experience. It is arguable that much of the bad press psychedelics have received in the decades since their vilification in the late 1960s has arisen as a result of negative psychedelic experiences in the context of poorly managed set and settings. When these factors are diligently managed, the vast majority of psychedelic experiences in most people are positive. The epidemiological work of Dr Teri Krebs, who looked at a very large sample of psychedelic users, illustrates the relative safety and benefit of psychedelic drug use in contemporary times. ${ }^{3}$

1 Sessa B, Johnson MW. Can psychedelic compounds play a part in drug dependence therapy? Br J Psychiatry 2015; 206: 1-3.

2 Sessa B. The Psychedelic Renaissance: Reassessing the Role of Psychedelic Drugs in the 21st Century Psychiatry and Society. Muswell Hill Press, 2012 p. 23.

3 Krebs TS, Johansen P. Psychedelics and mental health: a population study. PLOS One 2013; 8: e63972.

Dr Ben Sessa, Consultant Psychiatrist at AddAction and Senior Research Fellow at Cardiff University Medical School. Email: bensessa@gmail.com

doi: $10.1192 /$ bjp.206.5.434

\section{Are conclusions overstated for placebo response?}

The implications of Leuchter et al's research ${ }^{1}$ not only have potential for our further understanding of placebo responses in clinical trials, but also bring into question the pharmacological advantage of antidepressant medication over placebo in clinical outcomes for depression. Their findings warrant full evaluation so that they can be considered within the context of the wider research base. However, an accurate appraisal is currently limited by a lack of clarity in the methodology presented. We suggest several areas in which further clarification could assist critical appraisal.

First, the use of the Hamilton Rating Scale for Depression (HRSD) as a measure of depression severity warrants discussion. A 2014 literature review failed to find evidence to support its use, describing it as irretrievably flawed. Interestingly, many scale items were not found to sufficiently contribute to the measure of depression severity. ${ }^{2}$ Without a valid measure of severity, can we be assured that participants met criteria for at least moderate depressive symptoms at baseline? Any failure to exclude those with milder symptoms could also account for the similar outcomes demonstrated in pill-taking groups. The National Institute for Health and Care Excellence advocate the avoidance of antidepressant prescription in those with less than moderate depressive symptoms, because of the poor risk-benefit ratio. ${ }^{3}$

In terms of the study design, the sample size appears to be smaller than one would anticipate. This is not helped by the significant, $24 \%$ loss to follow-up. Given that the report does not reference a power calculation, are the authors able to provide clarity regarding their choice of sample size?

The process of recruitment also requires clarification. Recruitment via advertisement can be prone to selection bias and can account for loss of external validity within studies. ${ }^{4}$ We suggest that advertisement recruitment may have attracted participants particularly keen to seek active treatment, possibly in order to avoid healthcare expenditure. It is understood that random allocation of recruited participants took place. Further clarification regarding this process would be helpful.

It is also understood that research coordinators were blinded during supportive-care interactions. Double-blinding is clearly essential in a study that involves a subjective outcome measure. Given that the research coordinators were often trained nurses, we raise the concern that they may have recognised relevant side-effects and unintentionally deduced a participant's group assignment. With any loss of their impartiality, clinicians form expectations and these have the power to significantly influence outcomes. ${ }^{5}$ As trained nurses, it is also likely that their interactions might have provided therapeutic input aside from that considered to be consistent with supportive care. Were certain professionals more likely to report improvements in the placebo group?

Of further interest, we cannot find evidence to rule out suicidal behaviour as another potential confounder in this study. Participants' response to antidepressant medication may have been influenced by differences in serotonergic functioning, which has been linked to having a history of suicidal acts. ${ }^{6}$

With the above concerns in mind, we suggest that further consideration of the risk of type II error may be of value. We would be interested in the extent to which the authors have explored the potential for type II error and welcome their response.

1 Leuchter AF, Hunter AM, Tartter M, Cook IA. Role of pill-taking, expectation and therapeutic alliance in the placebo response in clinical trials for major depression. Br J Psychiatry 2014; 205: 443-9.

2 Bagby RM, Ryder AG, Schuller DR, Marshall MB. The Hamilton Depression Rating Scale: Has the gold standard become a lead weight? Am J Psychiatry 2014; 161: 2163-77. 
3 National Institute for Health and Clinical Excellence. Depression in Adults: The Treatment and Management of Depression in Adults (Clinical Guideline 90). National Institute for Health and Clinical Excellence, 2009

4 Fransen G, van Marrewijk C, Mujakovic S, Muris J, Laheij R, Numans M, et al. Pragmatic trials in primary care. Methodological challenges and solutions demonstrated by the DIAMOND-study. BMC Med Res Method 2007; 7: 16.

5 Even C, Siobud-Dorocant E, Dardennes RM. Critical approach to antidepressant trials. Blindness protection is necessary, feasible and measurable. Br J Psychiatry 2000; 177: 47-51.

6 Sullivan G, Oquendo M, Milak M, Miller J, Burke A, Ogden R, et al. Positron emission tomography quantification of serotonin $1 \mathrm{~A}$ receptor binding in suicide attempters with major depressive disorder. JAMA Psychiatry 2015; 72: $169-78$.

Bryony R. Corbyn, Psychiatry CT3, Mukesh Kripalani, London Deanery.

Email: Bryony.Corbyn@nhs.net

doi: 10.1192/bjp.206.5.434a

Author's reply: Dr Corbyn and Dr Kripalani's statement that our report 'brings into question the pharmacological advantage of antidepressant medication over placebo' is not warranted because our study was designed only to elucidate factors contributing to the placebo response in clinical trials. High placebo response rates in major depressive disorder (MDD) commonly lead to 'failed' trials (i.e. no statistical difference between drug and placebo). ${ }^{1}$ The fact that the medications showed numerical but not statistically significantly greater efficacy than placebo therefore is not surprising. Corbyn \& Kripalani suggest that the lack of statistical difference could represent a type II error. They are correct that we had limited power to detect such a difference, but this is not an error per se because the study was neither designed nor powered to examine the question.

Prior work has suggested that medication might not offer greater benefits than placebo except in moderate to severe depression. ${ }^{2}$ Corbyn \& Kripalani question whether the symptom severity in our sample was adequate to test our hypotheses. They specifically question our use of the HRSD, which they describe as 'irretrievably flawed', and ask whether they 'can be assured that participants met criteria for at least moderate depressive symptoms. First, as stated above, our aim was not to compare the efficacy of medication and placebo, so this concern is not relevant to the conclusions of our report. Second, all participants had diagnoses of MDD established using a structured interview instrument (Mini-International Neuropsychiatric Interview). Third, while there is no perfect symptom rating scale, the HRSD is the most widely used in clinical trials and does have some advantages over other instruments. ${ }^{3}$ The required score of $>17$ ensured that all participants met a commonly used threshold for depression treatment trials.

Corbyn \& Kripalani also ask for clarification regarding our choice of sample size. The study was powered to test our primary hypotheses, and the adequacy of the sample size can be assessed in part through the effect sizes of the regression analyses presented in Table 3 (p. 447). Our analyses examining expectations as predictors of outcome yielded highly significant results.

Corbyn \& Kripalani also express concern that 'recruitment via advertisement can be prone to selection bias and account for loss of external validity within studies'. All recruitment methods may introduce selection bias by including only a subset of those with MDD. For example, recruiting participants from a clinic biases a sample towards those who are better equipped to seek conventional care and who want only bona fide medication treatment, as opposed to those who may face barriers in accessing a clinic and are willing to possibly receive placebo in a research study. Because advertising for participants is a widely employed method for treatment research in MDD, our findings are likely to be relevant to other treatment study populations.

Additionally, Corbyn \& Kriplani's raise questions about the effectiveness of the treatment blinding in this study. We cannot determine whether there was any interaction between treatment assignment and nurses' symptom ratings. It is important to note, however, that raters remained blinded to the primary measure of interest in our results (expectation of the effectiveness of medications). Because these were formed at baseline, there was no possible influence of the nurses on this measure. Furthermore, as Corbyn \& Kripalani point out, there was no significant difference in depression treatment outcomes between medication and placebo treatment. It therefore seems unlikely that imperfections in the blinding would have been a significant contributor to our results. They also question whether 'suicidal behaviour' may have confounded our study results. Participants with any significant suicidal ideation were excluded from this study because of the possibility of placebo treatment.

Our report identified a novel form of expectation that contributed to heterogeneity in response to placebo. Corbyn \& Kripalani's letter highlights the fact that the design of the clinical trial itself also may contribute to heterogeneity in outcome. Their analysis underscores the need for future studies to examine the role of expectations in placebo response to confirm our results.

1 Leuchter AF, Hunter AM, Tartter M, Cook IA. Role of pill-taking, expectation and therapeutic alliance in the placebo response in clinical trials for major depression. Br J Psychiatry 2014; 205: 443-9.

2 Fournier JC, DeRubeis RJ, Hollon SD, Dimidjian S, Amsterdam JD, Shelton RC, et al. Antidepressant drug effects and depression severity: a patient-level meta-analysis. JAMA 2010; 303: 47-53.

3 Bech P. Rating scales in depression: limitations and pitfalls. Dialogues Clin Neurosci 2006; 8: 207-15.

Andrew F. Leuchter, MD, Aimee M. Hunter, PhD, Molly Tartter, PhD, Ian A. Cook, MD, University of California - Los Angeles. Email: afl@ucla.edu

doi: 10.1192/bjp.206.5.435

\section{Improving assessment and treatment of physical health problems in people with severe mental illness: the case for a shared IT system}

The poor assessment and treatment of physical health problems in people with schizophrenia found by Crawford et al is sadly not surprising.

The methodological problems that became evident in the pilot phase mirrors the problems faced in practice - many trusts do not have up-to-date physical health monitoring and these must be requested from primary care. Clinicians in mental health services interested in getting this information and who want to actively take part in physical health assessment and treatment have to, like Crawford et al, write to the general practitioner (GP) requesting this information and hope for a timely response.

Out of hours, there is no simple way of checking current medication, physical health conditions and allergies: information that is readily available on primary care databases. Many clinicians in mental health spend considerable time contacting GP practices to request investigations and results. Conversely, GPs are often frustrated at not finding out about changes in management plans and psychotropic medication quickly enough. This system of care is not conducive to the urgent need to improve physical healthcare for this group of patients.

A shared IT platform for primary care and mental health services, with up-to-date information on physical health such as 